TITLE:

\title{
Multiple scattering and stop band characteristics of flexural waves on a thin plate with circular holes
}

\author{
$\operatorname{AUTHOR}(\mathrm{S}):$
}

Wang, Zuowei; Biwa, Shiro

\section{CITATION:}

Wang, Zuowei ...[et al]. Multiple scattering and stop band characteristics of flexural waves on a thin plate with circular holes. Journal of Sound and Vibration 2018, 416: 80-93

\section{ISSUE DATE:}

2018-03-03

URL:

http://hdl.handle.net/2433/234651

\section{RIGHT:}

(c) 2018. This manuscript version is made available under the CC-BY-NC-ND 4.0 license

http://creativecommons.org/licenses/by-nc-nd/4.0/; The full-text file will be made open to the public on 3 March 2020 in accordance with publisher's 'Terms and Conditions for Self-Archiving'., この論文は出版社版でありません。引用の際 には出版社版をご確認ご利用ください。; This is not the published version. Please cite only the published version. 


\title{
Multiple scattering and stop band characteristics of flexural waves on a thin plate with circular holes
}

\author{
Zuowei Wang $^{\text {a,b }}$, Shiro Biwa ${ }^{\text {b,1 }}$ \\ ${ }^{a}$ School of Mechano-Electronic Engineering, Xidian University, P.O. Box 188, Xi'an 710071, China \\ E-mail: wangzw@mail.xidian.edu.cn \\ b Department of Aeronautics and Astronautics, Graduate School of Engineering, Kyoto University, Katsura, \\ Nishikyo-ku, Kyoto 615-8540, Japan \\ E-mail: biwa@kuaero.kyoto-u.ac.jp \\ ${ }^{1}$ Corresponding author. Tel: +81 75383 3796, E-mail: biwa@kuaero.kyoto-u.ac.jp (S. Biwa)
}

\begin{abstract}
A numerical procedure is proposed for the multiple scattering analysis of flexural waves on a thin plate with circular holes based on the Kirchhoff plate theory. The numerical procedure is based on the wave function expansion of the exciting as well as scattered fields, and the boundary conditions at the periphery of holes are incorporated as the relations between the expansion coefficients of exciting and scattered fields. A set of linear algebraic equations with respect to the wave expansion coefficients of the exciting field alone is established by the numerical collocation method. To demonstrate the applicability of the procedure, the stop band characteristics of flexural waves are analyzed for different arrangements and concentrations of circular holes on a steel plate. The energy transmission spectra of flexural waves are shown to capture the detailed features of the stop band formation of regular and random arrangements of holes. The increase of the concentration of holes is found to shift the dips of the energy transmission spectra toward higher frequencies as well as deepen them. The hexagonal hole arrangement can form a much broader stop band than the square hole arrangement for flexural wave transmission. It is also demonstrated that random arrangements of holes make the transmission spectrum more complicated.
\end{abstract}

Keywords: Flexural wave; Thin plate; Multiple scattering; Stop band formation; Kirchhoff plate theory 


\section{Introduction}

Propagation characteristics of flexural waves on thin plates have been studied extensively in the foregoing literature for their significant implications in the design of engineering structures and equipments. The interaction of flexural waves with different types of inclusions in plate structures, i.e., holes (cutouts), patches, inhomogeneities with different material properties or thicknesses, has been of particular interest regarding the dynamic stress concentration around them leading to possible structural failures [1] and the damage detection using elastic guided waves, e.g. [2]. Furthermore, when these inclusions are distributed on a plate, the overall propagation characteristics of flexural waves are altered. Recently, the phononic or metamaterial plate design by introducing artificial arrangements of inclusions is attracting increasing attention [3].

Problems of flexural wave scattering by a single inclusion have been studied by many authors. While the exact analysis of such problems needs to be carried out based on the threedimensional theory of elastodynamics, approximate plate theories have also been employed. Since the work of Pao and Chao [4] who analyzed the flexural wave scattering by a circular cavity, the Mindlin theory has been adopted by many other investigators [5-8]. Furthermore, when a plate is sufficiently thin, the classical Kirchhoff plate theory can be used to a good approximation to analyze the flexural wave scattering by inclusions of various types [9-13]. The validity of the results predicted by the Kirchhoff plate theory has been demonstrated by comparison with the results of the exact theory [11] and with experiments [14].

In contrast to the problems of flexural wave scattering by a single inclusion, the corresponding studies for multiple inclusions are more complicated and relatively scarce in the literature. The foregoing works include those of Lee and Chen using the null-field integral equation method [15] and the multipole method [16], although the numerical examples demonstrated by them are limited to the scattering by two inclusions. Peng [17] applied the so-called acoustic wave propagator method to analyze the multiple scattering of a flexural wave by nine cylindrical patches on a plate. Some investigators have analyzed the averaged propagation characteristics of flexural waves due to multiple scattering by random distributions of inclusions. Namely, Weaver [18] analyzed the multiple scattering of the flexural wave by random sprung masses. Dixon and Squire [19] analyzed the energy transport velocity of the flexural wave on a random plate with circular inclusions. Parnell and Martin [20] obtained the effective wavenumber of the flexural wave for a plate with random inclusions based on the quasi-crystalline approximation. On the other hand, when the inclusions are arranged periodically, the plate can exhibit so-called bandgaps or stop bands 
for the flexural wave propagation. Movchan et al. [21] analyzed the Bloch-Floquet waves in a plate with a periodic array of circular holes, and demonstrated the dispersion relation of the flexural wave including the bandgaps. Recently, Cai and Hambric [22] analyzed the multiple scattering of the flexural wave by 21 circular inclusions arranged on a square lattice and the corresponding stop band formation. The works mentioned above [15-22] were all based on the Kirchhoff plate theory.

As mentioned above, the understanding of the multiple scattering and the stop band formation of flexural waves is important for the design of phononic or metamaterial plates as it opens a way to the suppression, guiding, focusing, etc, of flexural waves. In the ideal case of an infinitely extended periodic array of inclusions, the flexural wave propagation is either allowed (pass band) or forbidden (stop band) depending on the frequency, according to the Bloch-Floquet theory [21]. In realistic situations, it is often necessary to analyze the stop band formation by a finite number of inclusions arranged on a plate. For periodic microstructures which are extended over a finite length in the propagation direction, the wave transmission spectrum shows complicated oscillatory features in addition to forming stop bands, as demonstrated for the wave transmission in multilayered structures [23] and fiberreinforced media [24]. Therefore, a complete understanding of stop band phenomena of flexural waves necessitates a numerical method for the multiple scattering analysis which can account for a large number of inclusions arranged on the plate in an arbitrary manner.

In this paper, a general numerical procedure is presented to analyze the multiple scattering of flexural waves on a thin plate with arbitrary arrangements of circular holes based on the Kirchhoff plate theory. The time-harmonic deflection field of the plate is given as the sum of the incident wave and the scattered waves by the holes, which are expressed by the wave function expansions used in other foregoing works, e.g. [22]. Instead of using the commonly employed expressions of the so-called Graf addition theorem, the present procedure employs the numerical collocation technique to construct a linear system of algebraic equations to determine the expansion coefficients directly, by following the computational multiple scattering studies for fiber-reinforced composites [25, 26]. Furthermore, the periodicity of the hole arrangement is assumed perpendicular to the propagation direction of the incident wave in order to save computational costs. Using the proposed method, the multiple scattering and the stop band formation of flexural wave is analyzed for different arrangements of circular holes with different concentrations. 


\section{Formulation}

\subsection{Governing equations of flexural motions of a thin plate}

Consider a thin, infinitely extended, isotropic and linear elastic plate containing $N$ nonoverlapping, through-thickness, circular holes (radius $R$ ) which are arranged arbitrarily in the $x-y$ plane as shown in Fig. 1. The position vector of an arbitrary point on the plate is denoted by $\mathbf{r}$, and the position vector of the center of the $l$ th hole $(l=1,2, \ldots, N)$ is denoted by $\mathbf{r}_{l}$. According to the Kirchhoff plate theory, the transverse motion of a thin plate without external loads is governed by [27]

$$
D \nabla^{4} w+\rho h \frac{\partial^{2} w}{\partial t^{2}}=0,
$$

where $w$ is the transverse displacement (deflection) of the plate, $t$ is the time, $h$ is the plate thickness, $\rho$ is the mass density, $D=E h^{3} /\left\{12\left(1-v^{2}\right)\right\}$ is the flexural rigidity of the plate, $E$ is Young's modulus, and $v$ is Poisson's ratio, respectively. The two-dimensional biharmonic operator is defined by $\nabla^{4}=\nabla^{2} \nabla^{2}$, where $\nabla^{2}$ is the Laplacian operator. A timeharmonic solution of Eq. (1) of a form $w=W e^{-\mathrm{i} \omega t}$ is considered in this paper, where $W$ is a complex-valued function of the position, $\mathrm{i}$ is the imaginary unit and $\omega$ is the angular frequency. The function $W$ satisfies the bi-Helmholtz equation

$$
\nabla^{4} W-k^{4} W=0,
$$

where $k$ is the wavenumber of the flexural wave given by

$$
k^{2}=\sqrt{\frac{\omega^{2} \rho h}{D}} .
$$

The solution of Eq. (2) can be expressed as the sum of the propagating part $W_{\mathrm{p}}$ and the

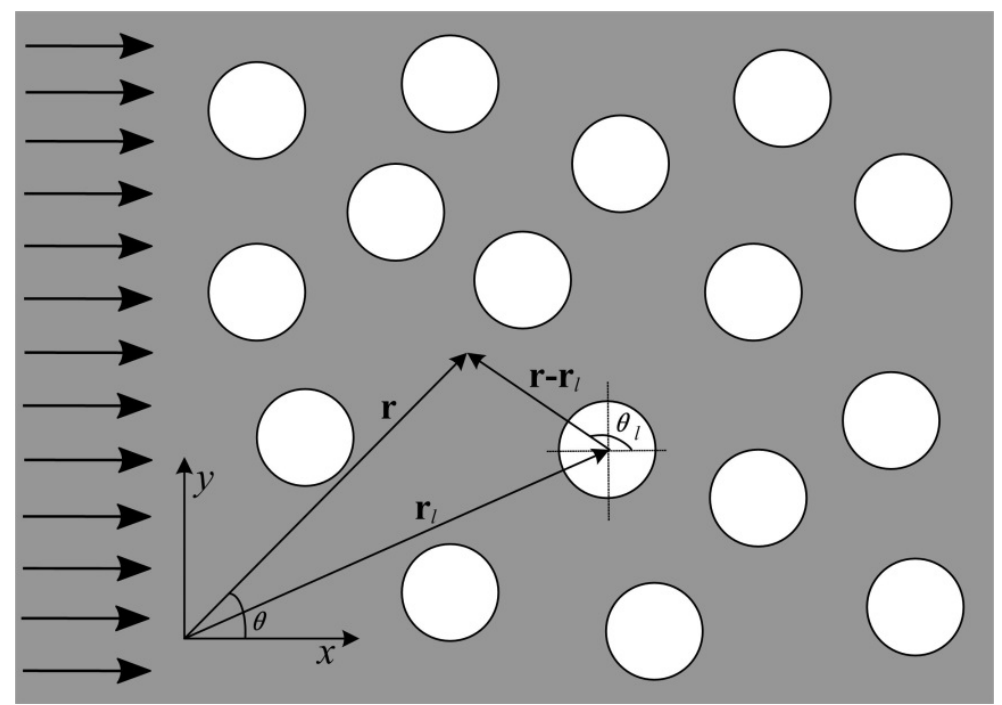

Figure 1. A thin plate with multiple holes subjected to the plane incident wave. 
attenuating part $W_{\mathrm{a}}$, i.e., $W=W_{\mathrm{p}}+W_{\mathrm{a}}$ [20-22]. They satisfy the Helmholtz equation and the modified Helmholtz equation, respectively,

$$
\begin{aligned}
& \nabla^{2} W_{\mathrm{p}}+k^{2} W_{\mathrm{p}}=0, \\
& \nabla^{2} W_{\mathrm{a}}-k^{2} W_{\mathrm{a}}=0 .
\end{aligned}
$$

By solving the above equations in the polar coordinate system, the displacement field can be written in a set of complete wave functions as [9]

$$
\begin{aligned}
& W_{\mathrm{p}}=\sum_{n=-\infty}^{\infty}\left(c_{n}^{1} J_{n}(k|\mathbf{r}|)+c_{n}^{3} H_{n}^{(1)}(k|\mathbf{r}|)\right) \mathrm{e}^{\mathrm{i} n \theta}, \\
& W_{\mathrm{a}}=\sum_{n=-\infty}^{\infty}\left(c_{n}^{2} I_{n}(k|\mathbf{r}|)+c_{n}^{4} K_{n}(k|\mathbf{r}|)\right) \mathrm{e}^{\mathrm{i} n \theta},
\end{aligned}
$$

where $\theta$ denotes the polar angle in the coordinate system centered at the origin of the Cartesian coordinates in Fig.1. In the above expressions, $H_{n}^{(1)}=J_{n}+\mathrm{i} Y_{n}$ is the $n$ th-order Hankel function of the first kind, $J_{n}$ and $Y_{n}$ are the $n$ th-order Bessel functions of the first and the second kind, $I_{n}$ and $K_{n}$ are the $n$ th-order modified Bessel functions of the first and the second kind, respectively, and $c_{n}^{1}, c_{n}^{2}, c_{n}^{3}, c_{n}^{4}$ are the complex expansion coefficients. The terms containing the functions $J_{n}$ and $I_{n}$ are finite at the origin $\mathbf{r}=\mathbf{0}$ in the polar coordinates.

On the other hand, the terms containing the functions $H_{n}^{(1)}$ and $K_{n}$ are singular at $\mathbf{r}=\mathbf{0}$. When $|\mathbf{r}| \rightarrow \infty$, the terms with $H_{n}^{(1)}$ represent outward-radiating waves and those with $K_{n}$ represent attenuating fields which decay exponentially in space.

\subsection{Multiple scattering of flexural waves}

The multiple scattering of flexural waves has been formulated by foregoing investigators in different manners [18-22]. In the present analysis, the formulation is given below in a manner commonly used for multiple scattering of waves in fiber reinforced media, e.g. [25, 26]. The incident wave is assumed to be a non-attenuating plane flexural wave propagating in the positive direction of the $x$-axis, which has the amplitude $W_{0}$ and the wavenumber $k$ satisfying Eq. (3). The deflection of the incident wave is given by

$$
W^{\text {inc }}(\mathbf{r})=W_{\mathrm{p}}^{\text {inc }}(\mathbf{r})=W_{0} \exp (\mathrm{i} k x) .
$$

The incident wave given by Eq. (6) is a solution of Eq. (4a) in the absence of holes. When the holes are present, the multiple scattering of the flexural wave occurs among them, and the scattered wave from each hole contains both propagating and attenuating parts. The propagating and attenuating parts of the deflection field scattered by the $l$ th hole $(l=$ 
$1,2, \cdots, N)$ can be expressed as the wave function expansion of Eq. (5) which are outwardradiating and decaying as $\left|\mathbf{r}-\mathbf{r}_{l}\right| \rightarrow \infty$, respectively, i.e.,

$$
\begin{aligned}
& W_{\mathrm{p}}^{l, \text { sca }}(\mathbf{r})=\sum_{n=-\infty}^{\infty} A_{n}^{l} H_{n}^{(1)}\left(k\left|\mathbf{r}-\mathbf{r}_{l}\right|\right) \mathrm{e}^{\mathrm{i} n \theta_{l}}, \\
& W_{\mathrm{a}}^{l, \text { sca }}(\mathbf{r})=\sum_{n=-\infty}^{\infty} B_{n}^{l} K_{n}\left(k\left|\mathbf{r}-\mathbf{r}_{l}\right|\right) \mathrm{e}^{\mathrm{i} n \theta_{l}},
\end{aligned}
$$

where $A_{n}^{l}$ and $B_{n}^{l}(n=0, \pm 1, \pm 2, \cdots ; l=1,2, \cdots, N)$ are the unknown expansion coefficients of the scattered deflection field, and $\theta_{l}$ denotes the polar angle in the coordinate system with the origin at the center of the $l$ th hole $\mathbf{r}=\mathbf{r}_{l}$ as shown in Fig.1.

The wave field in the neighborhood of the $l$ th hole, which excites that hole and creates the above scattered field, is referred to as the exciting field for that hole. The propagating and the attenuating parts of the exciting field for the $l$ th hole are the sum of the corresponding parts of the incident wave and the scattered waves from all the other holes as

$$
\begin{aligned}
& W_{\mathrm{p}}^{l, \text { exc }}(\mathbf{r})=W_{\mathrm{p}}^{\text {inc }}(\mathbf{r})+\sum_{j=1, j \neq l}^{N} W_{\mathrm{p}}^{j, \mathrm{sca}}(\mathbf{r}), \\
& W_{\mathrm{a}}^{l, \text { exc }}(\mathbf{r})=\sum_{j=1, j \neq l}^{N} W_{\mathrm{a}}^{j, \mathrm{sca}}(\mathbf{r}) .
\end{aligned}
$$

As the exciting field for the $l$ th hole is a solution of Eq. (4) in the absence of that hole [25], it should be finite at $\mathbf{r}=\mathbf{r}_{l}$. Therefore, it can be expressed in terms of the wave function expansion with the terms which are nonsingular at $\mathbf{r}=\mathbf{r}_{l}$, i.e.,

$$
\begin{aligned}
& W_{\mathrm{p}}^{l, \mathrm{exc}}(\mathbf{r})=\sum_{n=-\infty}^{\infty} E_{n}^{l} J_{n}\left(k\left|\mathbf{r}-\mathbf{r}_{l}\right|\right) \mathrm{e}^{\mathrm{i} n \theta_{l}}, \\
& W_{\mathrm{a}}^{l, \mathrm{exc}}(\mathbf{r})=\sum_{n=-\infty}^{\infty} F_{n}^{l} I_{n}\left(k\left|\mathbf{r}-\mathbf{r}_{l}\right|\right) \mathrm{e}^{\mathrm{i} n \theta_{l}},
\end{aligned}
$$

where $E_{n}^{l}$ and $F_{n}^{l}(n=0, \pm 1, \pm 2, \cdots ; l=1,2, \cdots, N)$ are the unknown wave expansion coefficients of the exciting field. From Eqs. (8) and (9), we obtain the following relations incorporating the coefficients of both exciting and scattered fields as

$$
\begin{gathered}
\sum_{n=-\infty}^{\infty} E_{n}^{l} J_{n}\left(k\left|\mathbf{r}-\mathbf{r}_{l}\right|\right) \mathrm{e}^{\mathrm{i} n \theta_{l}}=W_{\mathrm{p}}^{\mathrm{inc}}(\mathbf{r})+\sum_{j=1, j \neq l}^{N} \sum_{m=-\infty}^{\infty} A_{m}^{j} H_{m}^{(1)}\left(k\left|\mathbf{r}-\mathbf{r}_{j}\right|\right) \mathrm{e}^{\mathrm{i} m \theta_{j}}, \\
\sum_{n=-\infty}^{\infty} F_{n}^{l} I_{n}\left(k\left|\mathbf{r}-\mathbf{r}_{l}\right|\right) \mathrm{e}^{\mathrm{i} n \theta_{l}}=\sum_{j=1, j \neq l}^{N} \sum_{m=-\infty}^{\infty} B_{m}^{j} K_{m}\left(k\left|\mathbf{r}-\mathbf{r}_{j}\right|\right) \mathrm{e}^{\mathrm{i} m \theta_{j}}
\end{gathered}
$$


The displacement field in the neighborhood of the $l$ th hole is the sum of the exciting and the scattered fields, given by

$$
\begin{aligned}
W(\mathbf{r}) & =\sum_{n=-\infty}^{\infty}\left(A_{n}^{l} H_{n}^{(1)}\left(k\left|\mathbf{r}-\mathbf{r}_{l}\right|\right)+B_{n}^{l} K_{n}\left(k\left|\mathbf{r}-\mathbf{r}_{l}\right|\right)+E_{n}^{l} J_{n}\left(k\left|\mathbf{r}-\mathbf{r}_{l}\right|\right)\right. \\
& \left.+F_{n}^{l} I_{n}\left(k\left|\mathbf{r}-\mathbf{r}_{l}\right|\right)\right) \mathrm{e}^{\mathrm{i} \theta_{l}} .
\end{aligned}
$$

At the circular boundary of the $l$ th hole, the radial components of the bending moment and shear force should vanish, i.e.,

$$
\begin{aligned}
& M(\mathbf{r})=0, \quad\left|\mathbf{r}-\mathbf{r}_{l}\right|=R, \quad 0<\theta_{l}<2 \pi, \\
& V(\mathbf{r})=0, \quad\left|\mathbf{r}-\mathbf{r}_{l}\right|=R, \quad 0<\theta_{l}<2 \pi,
\end{aligned}
$$

The explicit expressions for the bending moment and shear force of thin plates are given by

$$
\begin{aligned}
& M(\mathbf{r})=-D\left[\frac{\partial^{2} W}{\partial\left|\mathbf{r}-\mathbf{r}_{l}\right|^{2}}+v\left(\frac{1}{\left|\mathbf{r}-\mathbf{r}_{l}\right|} \frac{\partial W}{\partial\left|\mathbf{r}-\mathbf{r}_{l}\right|}+\frac{1}{\left|\mathbf{r}-\mathbf{r}_{l}\right|^{2}} \frac{\partial^{2} W}{\partial \theta_{l}^{2}}\right)\right], \\
& V(\mathbf{r})=-D \frac{\partial\left(\nabla^{2} W\right)}{\partial\left|\mathbf{r}-\mathbf{r}_{l}\right|}-\frac{D(1-v)}{\left|\mathbf{r}-\mathbf{r}_{l}\right|^{2}} \frac{\partial}{\partial \theta_{l}}\left(\frac{\partial^{2} W}{\partial\left|\mathbf{r}-\mathbf{r}_{l}\right| \partial \theta_{l}}-\frac{1}{\left|\mathbf{r}-\mathbf{r}_{l}\right|} \frac{\partial W}{\partial \theta_{l}}\right) .
\end{aligned}
$$

Substituting Eq. (12) into the boundary conditions Eq. (13), the linear relations between the expansion coefficients $A_{n}^{l}, B_{n}^{l}$ and $E_{n}^{l}, F_{n}^{l}$ can be found for each azimuthal order $n=$ $0, \pm 1, \pm 2, \cdots$. Following Norris and Vemula [9], these relations are given by

$$
\begin{gathered}
{\left[\begin{array}{ll}
S_{H}(\kappa) & S_{K}(\kappa) \\
T_{H}(\kappa) & T_{K}(\kappa)
\end{array}\right]\left[\begin{array}{l}
A_{n}^{l} \\
B_{n}^{l}
\end{array}\right]=-\left[\begin{array}{ll}
S_{J}(\kappa) & S_{I}(\kappa) \\
T_{J}(\kappa) & T_{I}(\kappa)
\end{array}\right]\left[\begin{array}{c}
E_{n}^{l} \\
F_{n}^{l}
\end{array}\right],} \\
S_{X}(\kappa)=D\left[n^{2}(1-v) \mp \kappa^{2}\right] X_{n}(\kappa)-D(1-v) \kappa X_{n}^{\prime}(\kappa), \\
T_{X}(\kappa)=D n^{2}(1-v) X_{n}(\kappa)-D\left[n^{2}(1-v) \pm \kappa^{2}\right] \kappa X_{n}^{\prime}(\kappa),
\end{gathered}
$$

where $\kappa=k R$. In Eqs. (17) and (18), the upper sign is taken for $X=H^{(1)}$ or $J$, and the lower sign is taken for $X=I$ or $K$, respectively. The derivatives of the wave functions $H^{(1)}, J, I$ and $K$ can be calculated by using the following relations [28],

$$
\begin{gathered}
X_{n}^{\prime}(\kappa)=\frac{X_{n-1}(\kappa)-X_{n+1}(\kappa)}{2}, X=H^{(1)}, J \\
I_{n}^{\prime}(\kappa)=\frac{I_{n-1}(\kappa)+I_{n+1}(\kappa)}{2}, K_{n}^{\prime}(\kappa)=\frac{\mathrm{e}^{-\mathrm{i} \pi} K_{n-1}(\kappa)+\mathrm{e}^{\mathrm{i} \pi} K_{n+1}(\kappa)}{2} .
\end{gathered}
$$

By solving Eq. (16), a linear transformation matrix depending on the order $n$ but not on the position of the hole is obtained as

$$
\left[\begin{array}{c}
A_{n}^{l} \\
B_{n}^{l}
\end{array}\right]=\left[\begin{array}{ll}
M_{n}^{11} & M_{n}^{12} \\
M_{n}^{21} & M_{n}^{22}
\end{array}\right]\left[\begin{array}{c}
E_{n}^{l} \\
F_{n}^{l}
\end{array}\right],
$$

where the coefficients $M_{n}^{11}, M_{n}^{12}, M_{n}^{21}, M_{n}^{22}$ can be given explicitly as 


$$
\begin{aligned}
& M_{n}^{11}=-\frac{T_{K}(\kappa) S_{J}(\kappa)-S_{K}(\kappa) T_{J}(\kappa)}{S_{H}(\kappa) T_{K}(\kappa)-T_{H}(\kappa) S_{K}(\kappa)}, M_{n}^{12}=-\frac{T_{K}(\kappa) S_{I}(\kappa)-S_{K}(\kappa) T_{I}(\kappa)}{S_{H}(\kappa) T_{K}(\kappa)-T_{H}(\kappa) S_{K}(\kappa)}, \\
& M_{n}^{21}=-\frac{T_{J}(\kappa) S_{H}(\kappa)-S_{J}(\kappa) T_{H}(\kappa)}{S_{H}(\kappa) T_{K}(\kappa)-T_{H}(\kappa) S_{K}(\kappa)}, M_{n}^{22}=-\frac{T_{I}(\kappa) S_{H}(\kappa)-S_{I}(\kappa) T_{H}(\kappa)}{S_{H}(\kappa) T_{K}(\kappa)-T_{H}(\kappa) S_{K}(\kappa)}
\end{aligned}
$$

Then the coefficients $A_{n}^{l}$ and $B_{n}^{l}$ are given in terms of $E_{n}^{l}$ and $F_{n}^{l}$ as

$$
A_{n}^{l}=M_{n}^{11} E_{n}^{l}+M_{n}^{12} F_{n}^{l}, B_{n}^{l}=M_{n}^{21} E_{n}^{l}+M_{n}^{22} F_{n}^{l} .
$$

Substitution of Eq. (24) into Eqs. (10) and (11) leads to the following equations containing the expansion coefficients of the exciting field alone,

$$
\begin{aligned}
\sum_{n=-\infty}^{\infty} E_{n}^{l} J_{n}(k \mid \mathbf{r} & \left.-\mathbf{r}_{l} \mid\right) \mathrm{e}^{\mathrm{i} i \theta_{l}} \\
& =W_{\mathrm{p}}^{\mathrm{inc}}(\mathbf{r})+\sum_{j=1, j \neq l}^{N} \sum_{m=-\infty}^{\infty}\left(M_{m}^{11} E_{m}^{j}+M_{m}^{12} F_{m}^{j}\right) H_{m}^{(1)}\left(k\left|\mathbf{r}-\mathbf{r}_{j}\right|\right) \mathrm{e}^{\mathrm{i} m \theta_{j}}, \\
\sum_{n=-\infty}^{\infty} F_{n}^{l} I_{n}\left(k\left|\mathbf{r}-\mathbf{r}_{l}\right|\right) \mathrm{e}^{\mathrm{i} n \theta_{l}} & =\sum_{j=1, j \neq l}^{N} \sum_{m=-\infty}^{\infty}\left(M_{m}^{21} E_{m}^{j}+M_{m}^{22} F_{m}^{j}\right) K_{m}\left(k\left|\mathbf{r}-\mathbf{r}_{j}\right|\right) \mathrm{e}^{\mathrm{i} m \theta_{j}} .
\end{aligned}
$$

Once the expansion coefficients $E_{n}^{l}$ and $F_{n}^{l}$ are obtained, the deflection field on the plate is rewritten as

$$
\begin{aligned}
W(\mathbf{r}) & =W_{\mathrm{p}}^{\mathrm{inc}}(\mathbf{r})+\sum_{j=1}^{N} \sum_{m=-\infty}^{\infty}\left(M_{m}^{11} E_{m}^{j}+M_{m}^{12} F_{m}^{j}\right) H_{m}^{(1)}\left(k\left|\mathbf{r}-\mathbf{r}_{j}\right|\right) \mathrm{e}^{\mathrm{i} m \theta_{j}} \\
& +\sum_{j=1}^{N} \sum_{m=-\infty}^{\infty}\left(M_{m}^{21} E_{m}^{j}+M_{m}^{22} F_{m}^{j}\right) K_{m}\left(k\left|\mathbf{r}-\mathbf{r}_{j}\right|\right) \mathrm{e}^{\mathrm{i} m \theta_{j}}
\end{aligned}
$$

\section{Numerical implementation}

\subsection{Determination of expansion coefficients}

The purpose of the present study is to analyze the multiple scattering of flexural wave and the stop band phenomenon on an infinitely extended thin plate embedding a large number of holes. Specifically, the flexural wave transmission characteristics are analyzed when an infinite number of holes are arranged in the region $0<x<L$ and $-\infty<y<\infty$, where $L$ gives the length of the perforated region of the plate. In order to achieve this goal, it is assumed that the perforated region consists of a repetition of fundamental blocks in the $y$-direction as assumed in the foregoing numerical studies of multiple scattering in fiber-reinforced composites [25, 26], as shown in Fig. 2. Each fundamental block has $N_{\mathrm{f}}$ holes with an arbitrary arrangement. This assumption introduces the periodicity in the geometric layout of 
holes, and consequently the periodicity in the wave field, in the $y$-direction with the period $H$. This allows us to represent the scattered wave fields from infinite holes in terms of the expansion coefficients $E_{n}^{i}$ and $F_{n}^{i}$ for the $N_{\mathrm{f}}$ holes located within a single fundamental block, since the expansion coefficients are the same for the corresponding holes in all blocks. Then, Eqs. (25) and (26) can be reduced to the following forms involving the expansion coefficients for the $N_{\mathrm{f}}$ holes.

$$
\begin{aligned}
& \sum_{n=-\infty}^{\infty} E_{n}^{l} J_{n}\left(k\left|\mathbf{r}-\mathbf{r}_{l}\right|\right) e^{\mathrm{i} n \theta_{l}} \\
& =W_{\mathrm{p}}^{\text {inc }}(\mathbf{r})+\sum_{\substack{p=-\infty \\
p \neq 0}}^{\infty} \sum_{m=-\infty}^{\infty}\left(M_{m}^{11} E_{m}^{l}+M_{m}^{12} F_{m}^{l}\right) H_{m}^{(1)}\left(k\left|\mathbf{r}-\left(\mathbf{r}_{l}+p H \mathbf{e}_{2}\right)\right|\right) e^{\mathrm{i} m \theta_{l p}} \\
& +\sum_{p=-\infty}^{p=\infty} \sum_{\substack{j=1 \\
j \neq l}}^{N_{\mathrm{f}}} \sum_{m=-\infty}^{\infty}\left(M_{m}^{11} E_{m}^{j}+M_{m}^{12} F_{m}^{j}\right) H_{m}^{(1)}\left(k\left|\mathbf{r}-\left(\mathbf{r}_{j}+p H \mathbf{e}_{2}\right)\right|\right) e^{\mathrm{i} m \theta_{j p}} \\
& \sum_{n=-\infty}^{\infty} F_{n}^{l} I_{n}\left(k\left|\mathbf{r}-\boldsymbol{r}_{l}\right|\right) e^{\mathrm{i} n \theta_{l}}=\sum_{\substack{p=-\infty \\
p \neq 0}}^{\infty} \sum_{m=-\infty}^{\infty}\left(M_{m}^{21} E_{m}^{l}+M_{m}^{22} F_{m}^{l}\right) K_{m}\left(k\left|\mathbf{r}-\left(\mathbf{r}_{l}+p H \mathbf{e}_{2}\right)\right|\right) e^{\mathrm{i} m \theta_{l p}} \\
& +\sum_{p=-\infty}^{p=\infty} \sum_{\substack{j=1 \\
j \neq l}}^{N_{\mathrm{f}}} \sum_{m=-\infty}^{\infty}\left(M_{m}^{21} E_{m}^{j}+M_{m}^{22} F_{m}^{j}\right) K_{m}\left(k\left|\mathbf{r}-\left(\mathbf{r}_{j}+p H \mathbf{e}_{2}\right)\right|\right) e^{\mathrm{i} m \theta_{j p}}
\end{aligned}
$$

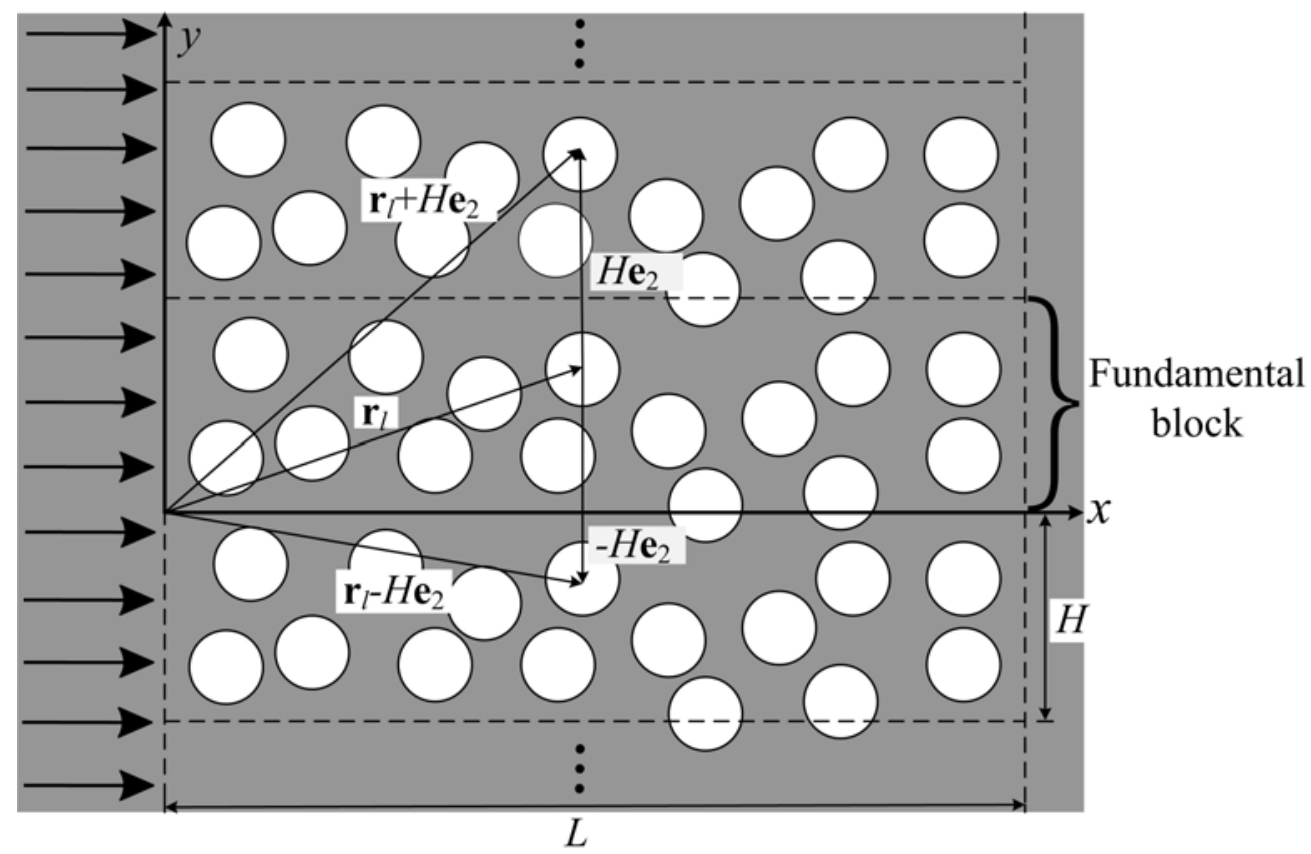

Figure 2. Numerical model consisting of the fundamental blocks. 
where $\mathbf{e}_{2}$ is the unit vector in the $y$-direction and $\theta_{l p}$ is the polar angle of a generic point $\mathbf{r}$ viewed from the center of the $l$ th hole in the $p$ th block $\left(l=1,2, \ldots, N_{\mathrm{f}}\right.$ and $\left.p=0, \pm 1, \pm 2, \cdots\right)$.

In order to calculate the expansion coefficients, the infinite series of wave function expansions in Eqs. (28) and (29) are truncated at a finite number for $n$ (or $m$ ) $=0, \pm 1$, $\pm 2, \cdots, \pm n_{\max }\left(=m_{\max }\right)$ and the analogous truncation is applied for the summation of the fundamental blocks for $p=0, \pm 1, \pm 2, \cdots, \pm p_{\max }$. The truncation parameters $n_{\max }$ and $p_{\max }$ should be carefully selected so that the further increase of their value makes negligible effects on the numerical solution. By this truncation, the number of unknown expansion coefficients is $N_{\mathrm{f}} \times\left(2 n_{\max }+1\right)$. In the present numerical analysis, $2 n_{\max }+2$ equidistant collocation points are selected on the boundary of each hole to establish a set of $N_{\mathrm{f}}\left(2 n_{\max }+2\right)$ equations to determine $N_{\mathrm{f}}\left(2 n_{\max }+1\right)$ coefficients. This over-determined system is in order to avoid the numerical problem of ill-conditioning of the coefficient matrix that could be observed in the numerical analysis of the set of linear equations. The constructed system of linear equations is solved by the matrix pseudoinverse [29].

\subsection{Numerical verification of energy conservation}

In order to verify the accuracy of the numerical results of the multiple scattering analysis, the energy conservation [9] is checked for the calculated flexural wave field on the thin plate. This is based on the principle which requires that the integral of time-averaged energy flux should vanish for a closed path on the plate enclosing no wave sources. In the present analysis, the expression for the energy flux density derived by Bobrovnitskii [30] is used.

For the present numerical analysis which uses the periodic arrangement of fundamental blocks, a rectangular path shown in Fig. 3 is chosen to evaluate if the energy conservation is

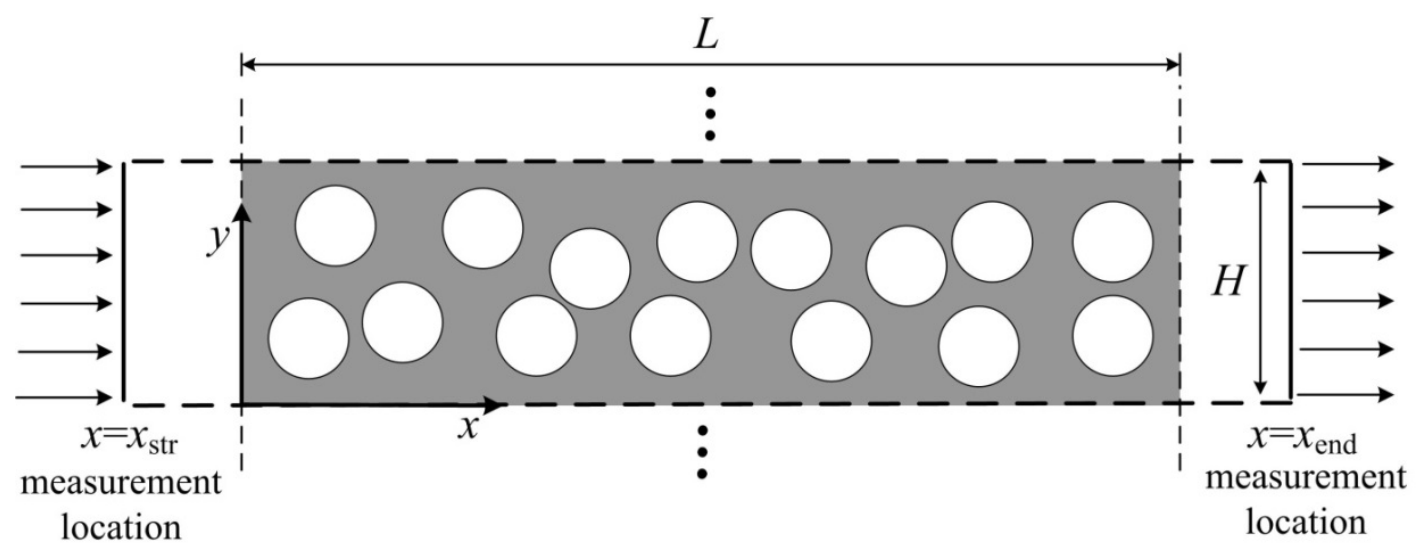

Figure 3. Rectangular integral path for verifying the energy conservation. 
satisfied accurately enough in the numerical solutions. The path has the same height as the fundamental block and the length is arbitrary defined by the two positions $x=x_{\text {end }}$ and $x=$ $x_{\text {str }}$. Since the sum of the net energy flux on the upper and the lower parts of the path vanishes due to the assumed periodicity in the $y$-direction, the principle of energy conversation is expressed as

$$
\frac{D \omega}{2} \int_{0}^{H} \operatorname{Im}\left[P\left(x_{\text {end }}, y\right)-P\left(x_{\text {str }}, y\right)\right] \mathrm{d} y=0,
$$

where

$$
P(x, y)=W \frac{\partial\left(\nabla^{2} \bar{W}\right)}{\partial x}-\frac{\partial W}{\partial x} \nabla^{2} \bar{W}+(1-v)\left(\frac{\partial W}{\partial x} \frac{\partial^{2} \bar{W}}{\partial y^{2}}-\frac{\partial W}{\partial y} \frac{\partial^{2} \bar{W}}{\partial x \partial y}\right)
$$

In the above expression, $\operatorname{Im}[\cdot]$ and $\overline{(\cdot)}$ denote the imaginary part and the complex conjugate of a complex variable, respectively.

The evaluation of the displacement field $W=W_{\mathrm{p}}+W_{\mathrm{a}}$ is straightforward after the expansion coefficients $E_{n}^{l}$ and $F_{n}^{l}$ are determined. The calculation of the derivatives such as $\partial W / \partial x$ can be performed by the transformation of the coordinate system

$$
\begin{aligned}
& \frac{\partial W}{\partial x}=\frac{\partial W}{\partial\left|\mathbf{r}-\mathbf{r}_{l}\right|} \cos \theta_{l}-\frac{\sin \theta_{l}}{\left|\mathbf{r}-\mathbf{r}_{l}\right|} \frac{\partial W}{\partial \theta_{l}}, \\
& \frac{\partial W}{\partial y}=\frac{\partial W}{\partial\left|\mathbf{r}-\mathbf{r}_{l}\right|} \sin \theta_{l}+\frac{\cos \theta_{l}}{\left|\mathbf{r}-\mathbf{r}_{l}\right|} \frac{\partial W}{\partial \theta_{l}} .
\end{aligned}
$$

Furthermore, as the functions $H_{n}^{(1)}$ and $K_{n}$ satisfy the Helmholtz equation Eq.(3) and the modified Helmholtz equation Eq.(4), respectively, $\nabla^{2} W$ can be simply replaced by

$$
\nabla^{2} W=\nabla^{2}\left(W_{\mathrm{p}}+W_{\mathrm{a}}\right)=k^{2}\left(-W_{\mathrm{p}}+W_{\mathrm{a}}\right),
$$

with the propagating part and the attenuating part of the displacement given by

$$
\begin{aligned}
& W_{\mathrm{p}}=W_{\mathrm{p}}^{\mathrm{inc}}+\sum_{p=-p_{\max }}^{p_{\max }} \sum_{j=1}^{N_{f}} \sum_{n=-n_{\max }}^{n_{\max }} A_{n}^{j} H_{n}^{(1)}\left(k\left|\mathbf{r}-\left(\mathbf{r}_{j}+p H \mathbf{e}_{2}\right)\right|\right) \mathrm{e}^{\mathrm{i} n \theta_{j}}, \\
& W_{\mathrm{a}}=\sum_{p=-p_{\max }}^{p_{\max }} \sum_{j=1}^{N_{f}} \sum_{n=-n_{\max }}^{n_{\max }} B_{n}^{j} K_{n}\left(k\left|\mathbf{r}-\left(\mathbf{r}_{j}+p H \mathbf{e}_{2}\right)\right|\right) \mathrm{e}^{\mathrm{i} n \theta_{j}} .
\end{aligned}
$$

\section{Numerical Examples}

In what follows, a steel plate containing circular holes is considered as the demonstrative examples of multiple scattering of flexural waves on thin plates. The incident wave is a plane flexural wave of unit amplitude propagating in the $x$-direction, and different incident wave frequencies are considered. The plate is assumed to have the thickness of $5 \mathrm{~mm}$ and contain 
holes with the same radius of $25 \mathrm{~mm}$. The material parameters of the plate are set as shown in Table 1.

\subsection{Preliminary analysis}

For the verification of the accuracy of the proposed method, a preliminary problem is considered for the fundamental block with four holes in a square arrangement. Numerical computations are performed with the truncation parameters of $n_{\max }=8$ and $p_{\max }=500$ (the selection of these parameters will be discussed later). The distance between the centers

Table 1 Material properties of the plate.

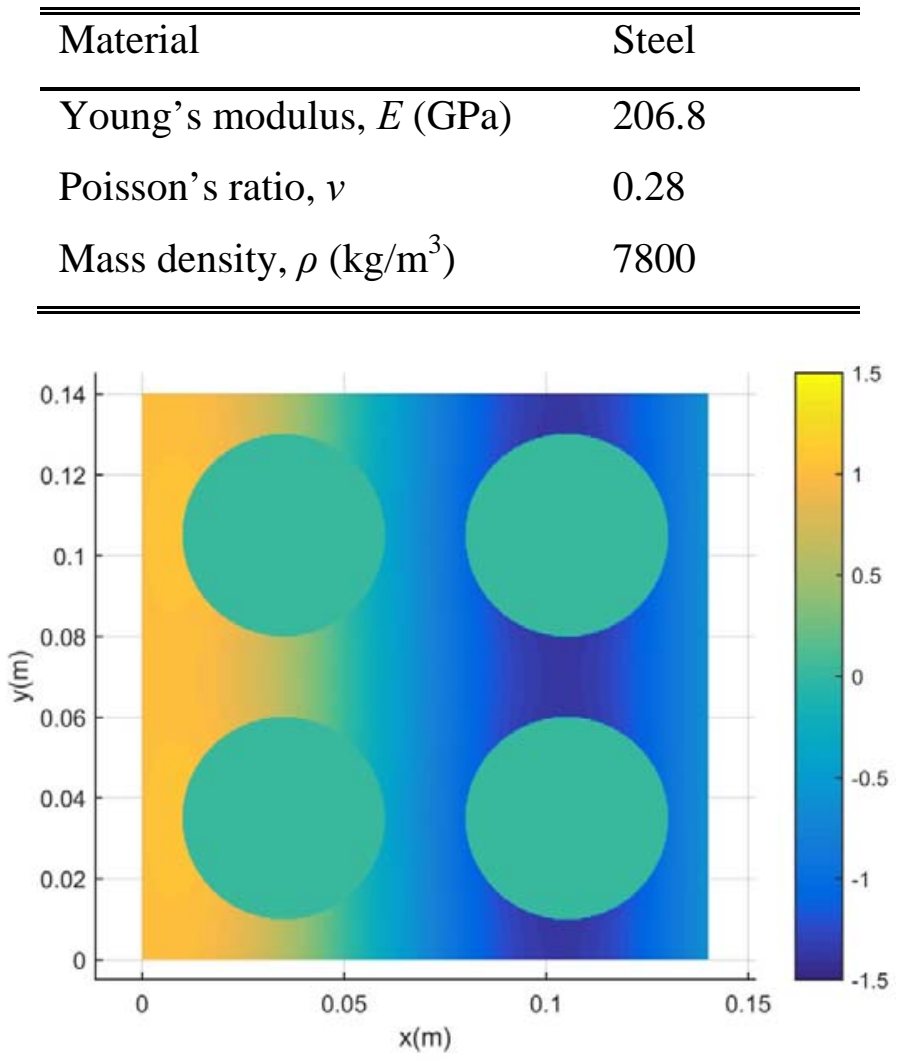

Figure 4. Deflection distribution in the fundamental block with four holes.

Table 2. Relative errors for energy conservation of multiple scattering analysis.

\begin{tabular}{llcl}
\hline \hline$f(\mathrm{~Hz})$ & $k R$ & $x_{\text {str }}=-0.07 \mathrm{~m}$, & $x_{\text {str }}=-0.112 \mathrm{~m}$, \\
& & $x_{\text {end }}=0.168 \mathrm{~m}$ & $x_{\text {end }}=0.28 \mathrm{~m}$ \\
\hline 1000 & 0.712 & $3.39 \times 10^{-4}$ & $-5.37 \times 10^{-4}$ \\
2000 & 1.007 & $-9.89 \times 10^{-5}$ & $-5.30 \times 10^{-6}$ \\
3000 & 1.233 & $-2.30 \times 10^{-4}$ & $-3.43 \times 10^{-4}$ \\
\hline \hline
\end{tabular}


of neighboring holes is set as $0.07 \mathrm{~m}$. Figure 4 shows the distribution of the real part of the complex-valued deflection $\operatorname{Re}[W]$ within the fundamental block subjected to the plane wave at frequency $f=1 \mathrm{kHz}$, corresponding to the normalized wavenumber $k R=0.712$. In order to check the numerical results, the relative error for the verification of energy conservation is defined by the ratio of the left-hand side of Eq. (30) to the energy flux of the incident wave for $0<y<H$. Two rectangular paths of the integral are chosen: $x_{\mathrm{str}}=-0.07 \mathrm{~m}$ and $x_{\mathrm{end}}=$ $0.168 \mathrm{~m} ; x_{\mathrm{str}}=-0.112 \mathrm{~m}$ and $x_{\mathrm{end}}=0.28 \mathrm{~m}$. Each vertical part of the integration path is divided by 20 integration points. For three different frequencies of the incident wave, the numerical results for the energy conservation are shown in Table 2. The nonzero relative errors are sufficiently small, which shows the effectiveness of numerical computations of the proposed method.

\subsection{Modeling of hole arrangements}

In order to examine the bandgap formation of flexural waves on the plate, different spatial arrangements of holes are assumed in the fundamental block: (i) square arrangement; (ii) hexagonal (triangular) arrangement; (iii) random arrangement, as shown in Fig. 5. In Fig. 5, $d$ is the distance between the centers of neighboring holes and $d_{0}$ is the distance between the

(a) Square arrangement

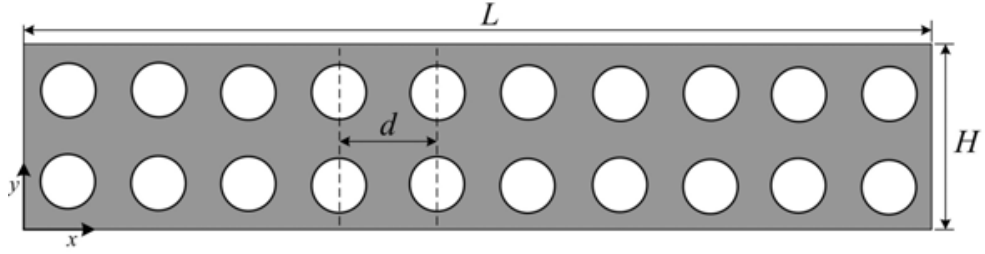

(b) Hexagonal arrangement

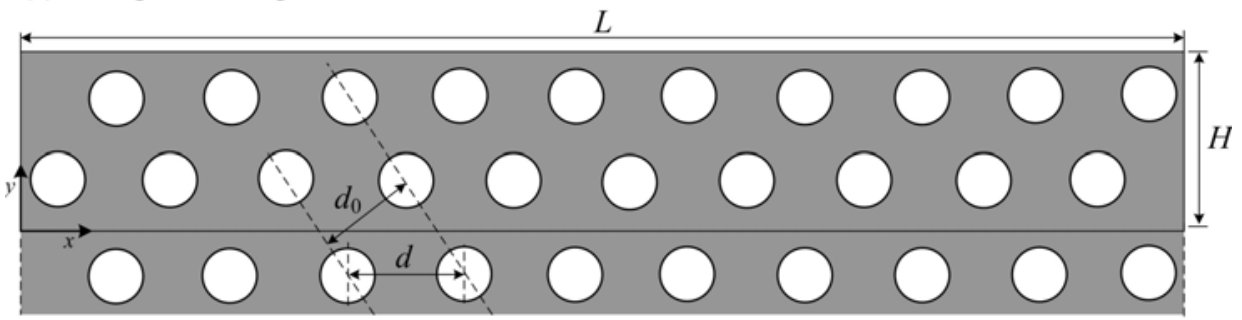

(c) Random arrangement

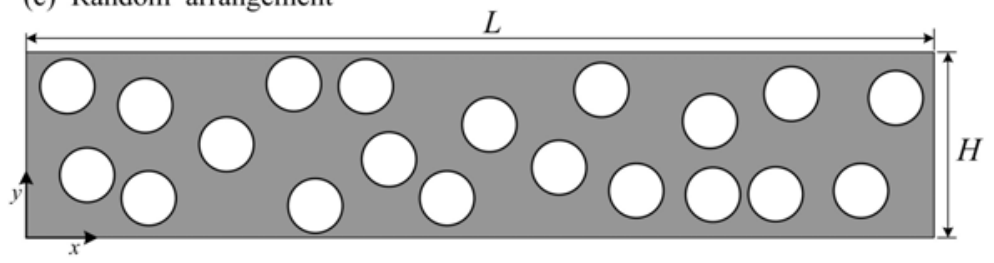

Figure 5. Hole arrangements for multiple scattering analysis of thin plate. 
parallel lines of the holes. The fundamental blocks for different hole arrangements have a fixed number of holes $N_{\mathrm{f}}=20$. The hole arrangements with different concentrations are constructed by changing the size of the fundamental block but keeping the number of holes and the ratio $L / H$ unchanged. The concentration of holes, denoted by $\phi$, is defined as the relative area of holes in the perforated region of the plate. For $\phi=0.4$, the length $L$ and height $H$ of the fundamental block for the three types of arrangements are given in Table 3 . For the random arrangements, the positions of holes are generated by the random sequential adsorption algorithm [31] for the specified concentration, with an additional condition that the neighboring holes are always separated by

$$
\left|\mathbf{r}_{l}-\mathbf{r}_{j}\right| \geq 2.01 R, l \neq j \text {. }
$$

The energy transmission coefficient is obtained as the ratio of the averaged energy flux density in the $x$-direction evaluated at $x=2 L$,

$$
\bar{P}_{x}=\frac{D \omega}{2 H} \int_{0}^{H} \operatorname{Im}[P(2 L, y)] \mathrm{d} y,
$$

to the time-averaged energy flux density of the incident wave $D \omega k^{3}$, where $P$ is defined in Eq. (31). The energy transmission coefficients are computed from $0.1 \mathrm{kHz}$ to $5.41 \mathrm{kHz}$, corresponding to the range of normalized wavenumber of the incident wave $0.225 \leq k R \leq$ 1.657.

\subsection{Selection of numerical truncation parameters}

When the frequency of the incident wave is sufficiently low and the wavelength is much larger than the radius of holes, the wave expansion of the displacement field requires only a few leading terms. As the wavelength becomes shorter (as the frequency becomes higher), an increasing number of terms (larger $n_{\text {max }}$ ) need to be considered in the analysis. The necessity of large $n_{\max }$ also depends on the distance between neighboring holes $d$. This is because the sharp gradients of the wave field will occur when the distance $d$ decreases to a certain extent.

Table 3 Dimensions of the fundamental block for $\phi=0.4$.

\begin{tabular}{lll}
\hline \hline & $L(\mathrm{~m})$ & $H(\mathrm{~m})$ \\
\hline Square arrangement & 0.70 & 0.14 \\
Hexagonal arrangement & 0.79 & 0.13 \\
Random arrangement & 0.70 & 0.14 \\
\hline \hline
\end{tabular}


For typical examples, Figs. 6 and 7 show the energy transmission coefficient for different concentrations of holes in square arrangement when different $n_{\max }$ are assumed while $p_{\max }=$ 500 is fixed. Two frequencies are arbitrarily chosen: $f=2.215 \mathrm{kHz}(k R=1.06)$ and $f=5 \mathrm{kHz}$

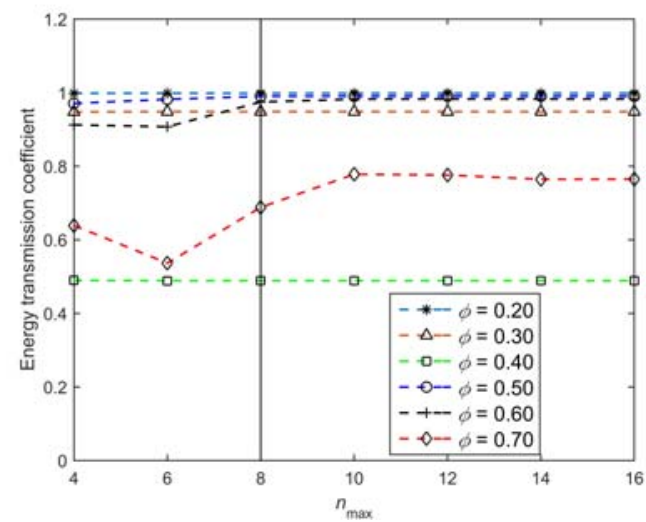

Figure 6. Energy transmission coefficient for different $n_{\max }$ for different concentrations of holes in square arrangement at $k R=1.06$.

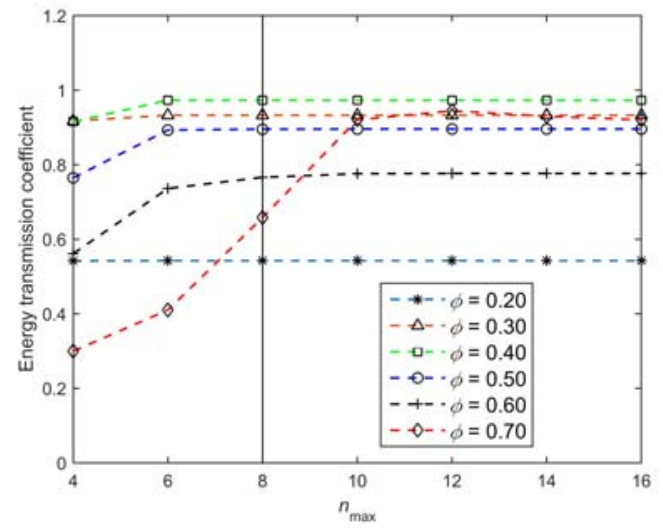

Figure 7. Energy transmission coefficient for different $n_{\max }$ for different concentrations of holes in square arrangement at $k R=1.596$.

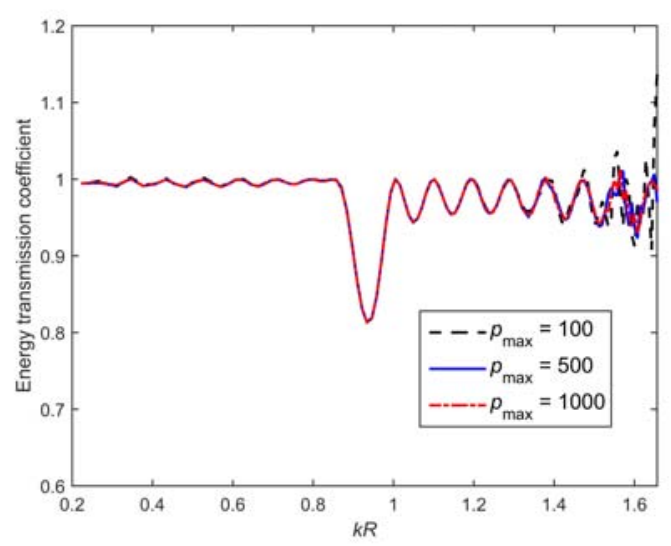

Figure 8. Energy transmission coefficient for different $p_{\max }$ for square hole arrangement with $\phi=0.3$. 
$(k R=1.596)$. As a result, it can be found that $n_{\max }=8$ is numerically sufficient for $\phi \leq$ 0.5 in the current frequency range. In the extreme case $\phi=0.7(d=53 \mathrm{~mm})$, a desirable value of $n_{\max }$ is expected to be as large as 16 .

The influence of truncating the sums for the repeated fundamental blocks in Eqs. (28)-(29) is also examined when $n_{\max }=8$ is fixed. Three cases for $p_{\max }=100,500$ and 1000 are examined for the square hole arrangement with $\phi=0.3$, as shown in Fig. 8. When $p_{\max }=$ 100 , it has enough numerical accuracy for the frequency range $k R \leq 1.473$ but the numerical result oscillates beyond the value of 1 as the frequency is further increased. This problem is eased by the increase of the value of $p_{\max }$. For the present numerical examples, $p_{\max }=500$ is used by considering both the satisfactory convergence and reasonably small computing cost.

\subsection{Results and discussion}

Figures 9 and 10 show the energy transmission spectra of flexural waves for square and hexagonal arrangements of holes with different concentrations. It is noted that the transmission spectra are presented here as the relation between the energy transmission coefficient and the normalized wavenumber of the incident wave. For the square arrangements of holes, the transmission spectra in Fig. 9 are found to have clear dips at different wavenumbers depending on the concentration of holes: the dip of the energy transmission spectra is shifted toward higher wavenumbers and deepened as the concentration of holes increases. In Fig. 10 for the hexagonal arrangements of holes, the transmission spectra exhibit much wider and deeper ranges of vanishing energy transmission than the corresponding square arrangements of holes. It is noted here that the energy transmission coefficient in Figs. 9 and 10 have values slightly exceeding 1 (by only a few percent) at some points near the edges of the dip. This is likely due to a numerical error by the truncation of infinite series in Eqs. (28) and (29), which could be improved by further increasing the parameter $p_{\max }$.

Figures 11 and 12 show the distribution of the deflection $\operatorname{Re}[W]$ in the fundamental block when the transmission coefficient is at the dip, i.e., at $f=2.215 \mathrm{kHz}(k R=1.06)$ for the square hole arrangement and at $f=3.97 \mathrm{kHz}(k R=1.42)$ for the hexagonal hole arrangement, respectively. In Fig. 11, the wave appears to be transmitted through the perforated region with the square hole arrangement with partial reduction of amplitude, as the energy transmission coefficient is about 0.5. On the other hand, the energy transmission of flexural waves appears to be totally blocked for the hexagonal hole arrangement in Fig. 12. 
When the plate has an infinitely periodic array of holes, it is known that the scattered waves from the holes interfere constructively and the energy transmission is forbidden in certain bands of the frequency (stop bands) [21]. A similar trend can be observed in the present results where the holes are arranged for a finite length in the propagation direction. The dips of the transmission coefficient correspond to the stop band. The energy transmission coefficient takes a nonzero but relatively small value at the dips in Fig. 9, while it appears to diminish in a certain band of frequency in Fig. 10. Moreover, it can be seen that the transmission coefficient shows oscillatory behavior with the wavenumber, more evidently in

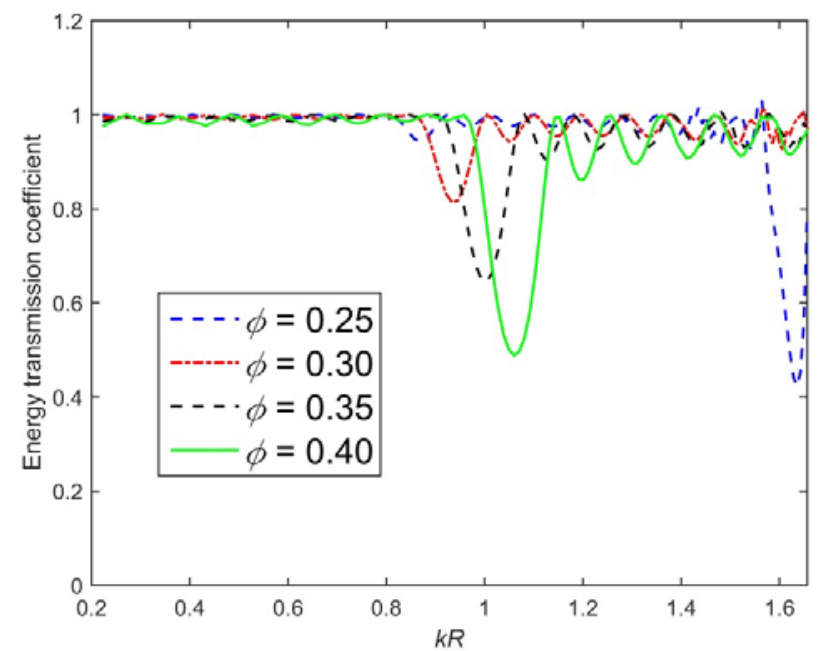

Figure 9. Energy transmission coefficients of flexural wave for square hole arrangements with different concentrations $\phi$.

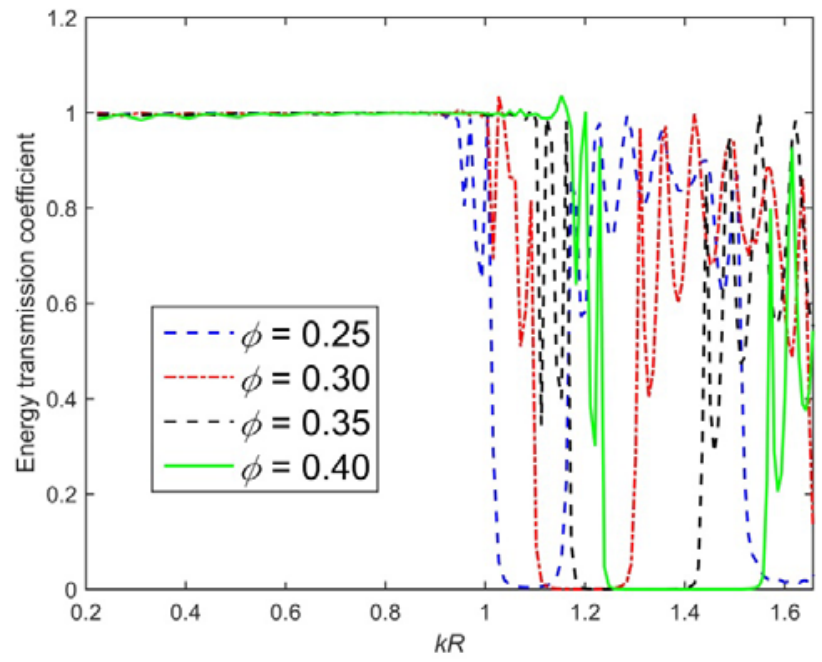

Figure 10. Energy transmission coefficients of flexural wave for hexagonal hole arrangements with different concentrations $\phi$. 
Fig. 9. These trends have also been observed in the analysis of wave transmission across multilayers or fiber arrangements extending over finite lengths [23, 24], where the number of oscillation cycles in the transmission spectrum and the depth of dips are related to the number of unit cells of the finite periodic structure.

Based on simple geometrical considerations, the condition for the above-mentioned stop bands can be given by [26]

Square arrangement: $\quad k^{\mathrm{eff}}=n \pi / d$,

Hexagonal arrangement: $\quad k^{\mathrm{eff}}=2 n \pi /\left(\sqrt{3} d_{0}\right)$,

where $k^{\mathrm{eff}}$ is the effective wavenumber of the perforated plate. For the spacing parameters $d$ and $d_{0}$ corresponding to different values of the hole concentration, the normalized effective wavenumber $k^{\mathrm{eff}} R$ is calculated by the right-hand side of Eqs. (38) and (39), and shown in Table 4. It is then observed that the calculated values of $k^{\mathrm{eff}} R$ are in fair agreement with the location of the first dips shown in Figs. 9 and 10. Naturally, the wavenumber of the perforated plate $k^{\text {eff }}$ differs from the wavenumber of the original homogenous plate $k$. Since the perforated plate is expected to have a slower velocity and a higher wavenumber, the

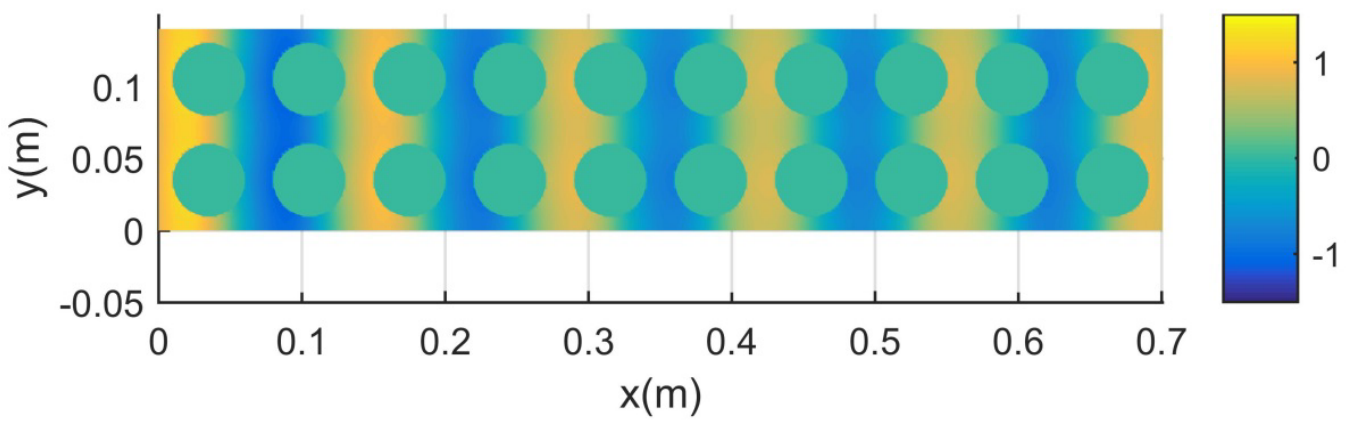

Figure 11. Deflection distribution in the fundamental block with square hole arrangement:

$$
=0.40 ; k R=1.06 \text {. }
$$

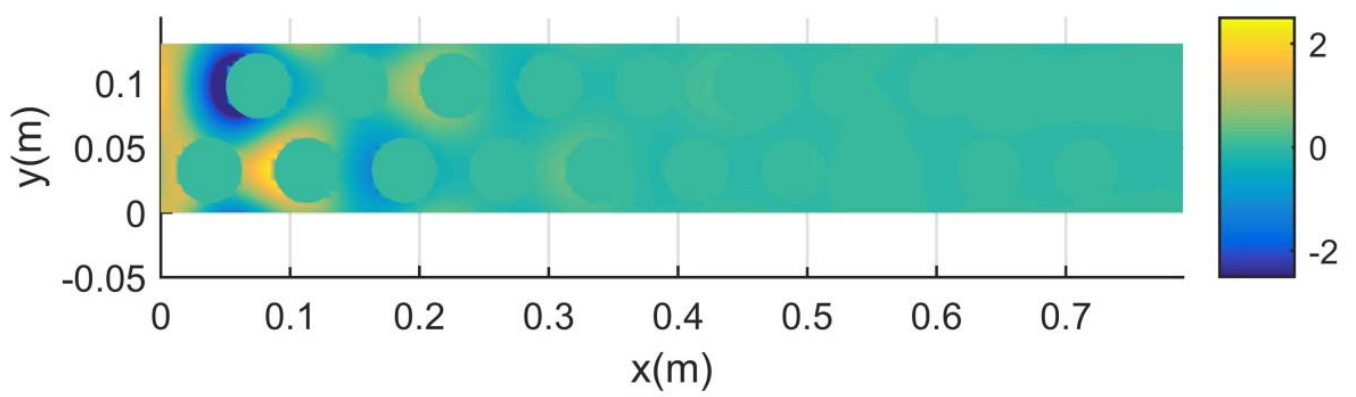

Figure 12. Deflection distribution in the fundamental block with hexagonal hole arrangement: $\phi=0.40 ; k R=1.42$. 
values of $k^{\text {eff }} R$ given in Table 4 should be larger than the locations of the dips along the wavenumber axis. This is clearly shown in Fig. 9 as the deviation of the value of $k^{\mathrm{eff}} R=0.178$ in Table 4 from the location of the second dip for the concentration $\phi=0.25$. On the other hand, the difference between the effective wavenumber $k^{\text {eff }}$ and the original wavenumber $k$ is small for the flexural wave with relatively long wavelengths on the perforated plate with relatively small hole concentrations, which makes Eqs. (38) and (39) a reasonable rough estimate for the location of the first stop band.

In order to examine the effect of perturbed hole arrangements on the flexural wave transmission, the simulations are also performed for four random arrangements of 20 holes in the fundament block in the case where $\phi=0.4$. The energy transmission spectra corresponding to four random arrangements are demonstrated in Fig. 13, together with the averaged transmission spectrum. It is noted here that the random arrangements of holes considered here have a finite length in the propagation direction and periodic in the vertical direction. Therefore, the present simulations do not correspond to the case of randomly distributed holes on an infinitely extended plate, and the four arrangements give different transmission characteristics. Compared with the regular arrangements, the random arrangements exhibit more complex energy transmission characteristics. As a matter of fact, the pass and stop band features observed for square and hexagonal arrangements tend to disappear for the random arrangements, and the transmission coefficient drops significantly in a much wider range of $k R$ for the plate with random arrangements of holes. This may be partly due to the local small spacings between neighboring holes, but further examinations are left for future investigations. For a relatively narrow dip identified for a single random arrangement (Pattern 4) in the frequency range between $k R=1.49$ and 1.59 , the deflection

Table 4 Estimated wavenumbers for stop bands for square and hexagonal arrangements.

\begin{tabular}{llllll}
\hline \hline \multicolumn{2}{l}{ Square arrangement } & \multicolumn{4}{l}{ Hexagonal arrangement } \\
\hline$\phi$ & $d(\mathrm{~m})$ & $k^{\mathrm{eff}} R$ & $\phi$ & $d(\mathrm{~m})$ & $k^{\text {eff }} R$ \\
\hline 0.25 & 0.089 & 0.089 & 0.25 & 0.095 & 1.010 \\
& & 0.178 & & & \\
0.30 & 0.081 & 0.970 & 0.30 & 0.087 & 1.205 \\
0.35 & 0.075 & 1.048 & 0.35 & 0.080 & 1.301 \\
0.4 & 0.070 & 1.120 & 0.4 & 0.075 & 1.390 \\
\hline \hline
\end{tabular}


amplitude distribution in the fundamental block is shown in Fig. $14(k R=1.56)$, which shows that a random arrangement also exhibits a strong filtering for flexural waves at special frequencies.

\section{Conclusion}

In this paper, a numerical procedure has been proposed for multiple scattering analysis of flexural waves on thin plates with circular holes based on the Kirchhoff plate theory. The proposed procedure is based on the wave function expansion of the scattered fields and the numerical collocation method to determine the expansion coefficients. The method is capable of analyzing arbitrary arrangements of holes and suitable for the stop band analysis of

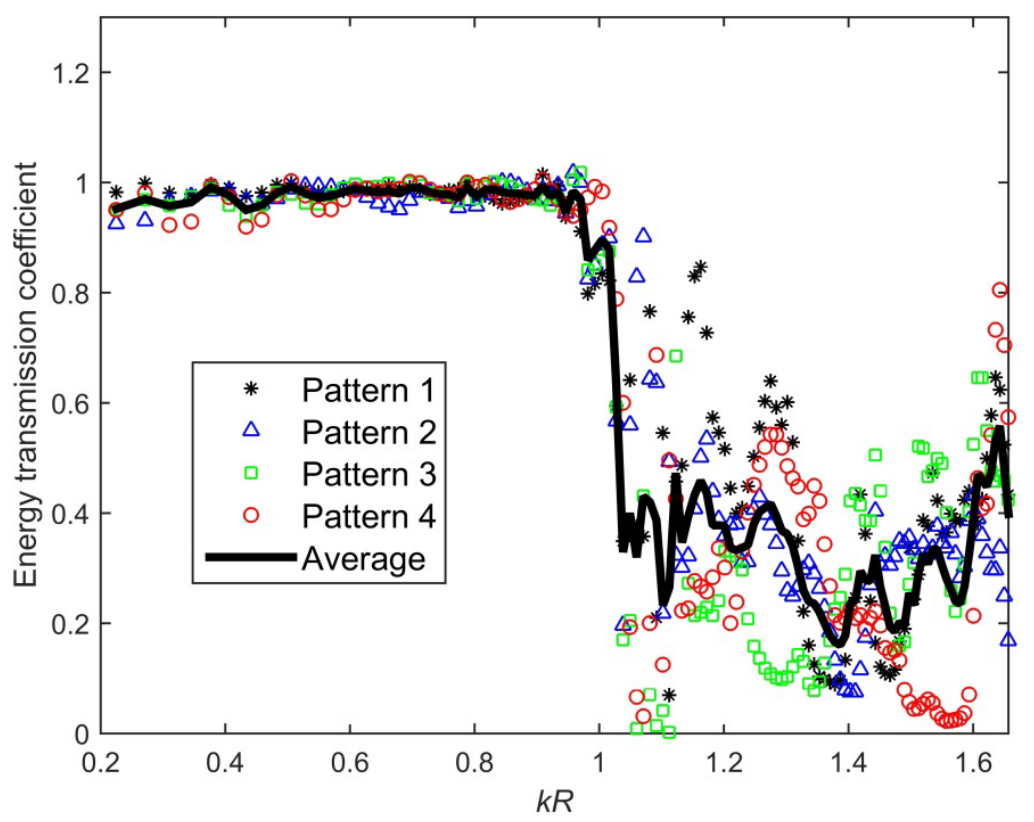

Figure 13. Energy transmission coefficients of flexural wave for different random arrangements for the case $\phi=0.4$.

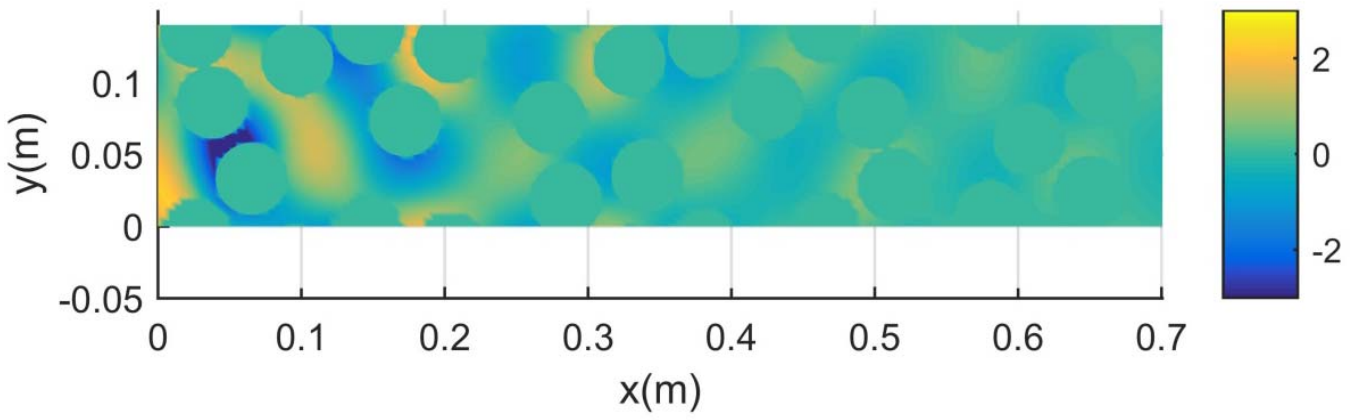

Figure 14. Deflection distribution in the fundamental block with $\phi=0.4$ and $k R=1.56$. 
flexural waves on thin plates containing a large number of holes. The stop band characteristics of flexural waves have been demonstrated for different arrangements and concentrations of holes. The energy transmission spectra of flexural waves exhibit the stop band formation for regular arrangements of holes. The transmission spectra for disordered hole arrangements have also been demonstrated showing more complicated features. The proposed numerical procedure can be easily extended for more general multiple scattering and bandgap analysis of flexural waves on thin plates with other types of inclusions.

\section{Acknowledgments}

This research is supported by the National Natural Science Foundation of China (Grant No. 51605360). Z. Wang acknowledges the financial support from the China Scholarship Council for his postdoctoral research in Kyoto University.

\section{References}

[1] Y.-H. Pao, C. C. Mow, Diffraction of Elastic Waves and Dynamic Stress Concentrations, Crane Russak, New York, 1973.

[2] L. R. F. Rose, C. H. Wang, Mindlin plate theory for damage detection: Imaging of flexural inhomogeneities, Journal of the Acoustical Society of America, 127 (2010), pp. 754-763.

[3] R. Zhu, X. N. Liu, G. K. Hu, F. G. Yuan, G. L. Huang, Microstructural designs of plate-type elastic metamaterial and their potential applications: a review, International Journal of Smart and Nano Materials, 6(1) (2015), pp.14-40.

[4] Y.-H. Pao, C. C. Chao, Diffractions of flexural waves by a cavity in an elastic plate, AIAA Journal, 2 (1964), pp. 2004-2010.

[5] R. Paskaramoorthy, A. H. Shah, S. K. Datta, Scattering of flexural waves by cavities in a plate, International Journal of Solids and Structures, 25 (1989), pp. 1177-1191.

[6] C. Vemula, A. N. Norris, Flexural wave propagation and scattering on thin plates using Mindlin theory, Wave Motion, 26 (1997), pp. 1-12.

[7] C. H. Wang, F.-K. Chang, Scattering of plate waves by a cylindrical inhomogeneity, Journal of Sound and Vibration, 282 (2005), pp. 429-451.

[8] F. B. Cegla, A. Rohde, M. Veidt, Analytical prediction and experimental measurement for mode conversion and scattering of plate waves at non-symmetric circular blind holes in isotropic plates, Wave Motion, 45 (2008), pp. 162-177.

[9] A. N. Norris, C. Vemula, Scattering of flexural waves on thin plates, Journal of Sound and Vibration, 118 (1995), pp. 115-125.

[10] V. A. Squire, T. W. Dixon, Scattering of flexural waves from a coated cylindrical anomaly in a thin plate, Journal of Sound and Vibration, 236 (2000), pp. 367-373. 
[11] T. Grahn, Lamb wave scattering from a circular partly through-thickness hole in a plate, Wave Motion, 37 (2003), pp. 63-80.

[12] V. V. Matus, V. F. Emets, T-matrix method formulation applied to the study of flexural waves scattering from a through obstacle in a plate, Journal of Sound and Vibration, 329 (2010), pp. 2843-2850.

[13] A. Climente, A. N. Norris, J. Sánchez-Dehesa, Scattering of flexural waves from a hole in a thin plate with an internal beam, Journal of the Acoustical Society of America, 137 (2015), pp293-302.

[14] P. Fromme, M. F. Sayir, Measurement of the scattering of a Lamb wave by a through hole in a plate, Journal of the Acoustical Society of America, 111 (2002), pp. 1165-1170.

[15] W. M. Lee, J. T. Chen, Scattering of flexural wave in a thin plate with multiple circular inclusions by using the null-field integral equation approach, Journal of Sound and Vibration, 329 (2010), pp. 1042-1061.

[16] W. M. Lee, J. T. Chen, Scattering of flexural wave in a thin plate with multiple circular inclusions by using the multipole method, International Journal of Mechanical Sciences, 53 (2011), pp. 617-627.

[17] S. Z. Peng, Flexural wave scattering and dynamic stress concentration in a heterogeneous plate with multiple cylindrical patches by acoustical wave propagator technique, Journal of Sound and Vibration, 286 (2005), pp. 729-743.

[18] R. L. Weaver, Multiple-scattering theory for mean responses in a plate with sprung masses, Journal of the Acoustical Society of America, 101 (1997), pp. 3466-3474.

[19] T. W. Dixon, V. A. Squire, Energy transport velocity of flexural waves in a random medium, Waves in Random Media, 10 (2000), pp. 83-102.

[20] W. J. Parnell, P. A. Martin, Multiple scattering of flexural waves by random configurations of inclusions in thin plates, Wave Motion, 48 (2011), pp. 161-175.

[21] A. B. Movchan, N. V. Movchan, R. C. McPhedran, Bloch-Floquet bending waves in perforated thin plates, Proceedings of the Royal Society, A463 (2007), pp. 2505-2518.

[22] L.-W. Cai, S. A. Hambric, Multiple scattering of flexural waves on thin plates, ASME Journal of Vibration and Acoustics, 138 (2016), 011009.

[23] Y. Ishii and S. Biwa, Ultrasonic evaluation of interlayer interfacial stiffness of multilayered structures, Journal of Applied Physics, 111 (2012), 084907.

[24] F. Kobayashi, S. Biwa, N. Ohno, Wave transmission characteristics in periodic media of finite length: multilayers and fiber arrays, International Journal of Solids and Structures, 41 (2004), pp. 7361-7375.

[25] S. Biwa, S. Yamamoto, F. Kobayashi, N. Ohno, Computational multiple scattering analysis for shear wave propagation in unidirectional composites, International Journal of Solids and Structures, 41 (2004), pp. 435-457.

[26] T. Sumiya, S. Biwa, G. Haïat, Computational multiple scattering analysis of elastic waves in unidirectional composites, Wave Motion, 50 (2013), pp. 253-270.

[27] K. F. Graff, Wave Motion in Elastic Solids, Oxford University Press, London, 1975.

[28] M. Abramowitz, I. A. Stegun, Handbook of Mathematical Functions with Formulas, Graphs, and Mathematical Tables, Dover Publications, New York, 1964.

[29] G. H. Golub, C. F. Van Loan, Matrix Computations, Johns Hopkins University Press, Baltimore, 1996. 
[30] Y. I. Bobrovnitskii, Calculation of the power flow in flexural waves on thin plates, Journal of Sound and Vibration, 194 (1996), pp.103-106.

[31] J. Feder, Random sequential adsorption, Journal of Theoretical Biology, 87 (1980), pp.237-254. 
Table and Figure Legends

Table 1 Material properties of the plate.

Table 2 Relative errors for energy conservation of multiple scattering analysis.

Table 3 Dimensions of the fundamental block for $\phi=0.4$.

Table 4 Estimated wavenumbers for stop bands for square and hexagonal arrangements.

Figure 1. A thin plate with multiple holes subjected to the plane incident wave.

Figure 2. Numerical model consisting of the fundamental blocks.

Figure 3. Rectangular integral path for verifying the energy conservation.

Figure 4. Deflection distribution in the fundamental block with four holes.

Figure 5. Hole arrangements for multiple scattering analysis of thin plate.

Figure 6. Energy transmission coefficient for different $n_{\max }$ for different concentrations of holes in square arrangement at $k R=1.06$.

Figure 7. Energy transmission coefficient for different $n_{\max }$ for different concentrations of holes in square arrangement at $k R=1.596$.

Figure 8. Energy transmission coefficient for different $p_{\max }$ for square hole arrangement with $\phi=0.3$.

Figure 9. Energy transmission coefficients of flexural wave for square hole arrangements with different concentrations $\phi$.

Figure 10. Energy transmission coefficients of flexural wave for hexagonal hole arrangements with different concentrations $\phi$.

Figure 11. Deflection distribution in the fundamental block with square hole arrangement: $=0.40 ; k R=1.06$.

Figure 12. Deflection distribution in the fundamental block with hexagonal hole arrangement: $=0.40 ; k R=1.42$.

Figure 13. Energy transmission coefficients of flexural wave for different random arrangements for the case $\phi=0.4$.

Figure 14. Deflection distribution in the fundamental block with $\phi=0.4$ and $k R=1.56$. 